\title{
Differential Spatial Modulation for APSK in Time-Varying Fading Channels
}

\author{
Philippa A. Martin, Senior Member, IEEE
}

\begin{abstract}
We develop a novel differential spatial modulation (DSM) scheme for amplitude phase shift keying (APSK) modulation, which can either improve throughput or performance over DSM for PSK. Then we investigate the impact of time-varying fading on DSM. We find performance degrades if the fading is too fast due to differential detection. The impact of a long outer error control code $(E C C)$ is also considered. Its performance is limited by the slowly varying channel required for differential detection. We consider using reconfigurable antennas to periodically change the channel conditions and hence significantly improve coded performance for DSM systems.
\end{abstract}

Index Terms-Spatial modulation, APSK, differential modulation, fading channel, reconfigurable antennas, LDPC code.

\section{INTRODUCTION}

Spatial modulation (SM) improves energy efficiency and reduces the number of RF chains required compared to multiple input multiple output (MIMO) transmission (by transmitting from one or a small subset of available antennas at any given time) [1], [2]. Differential SM (DSM) avoids the need for channel state information (CSI) at the transmitter or receiver, at the cost of performance. In [3], [4] a differential spatial modulation (DSM) approach was proposed for equal energy constellations, namely $M$-ary phase shift keying ( $M$ $P S K)$. The transmit antennas take turns transmitting $M$-PSK symbols, where each symbol is chosen using $\log _{2}(M)$ bits. In [4], $\left\lfloor\log _{2}\left(N_{t} !\right)\right\rfloor$ additional information bits are coded into the order that antennas are used with transmission defined over $N_{t}$ symbol periods, where $N_{t}$ is the number of transmit antennas. This increases the spectral efficiency to $\left(N_{t} \log _{2}(M)+\left\lfloor\log _{2}\left(N_{t} !\right)\right\rfloor\right) / N_{t}$ b/s/Hz. Another DSM approach was proposed for $M$-PSK in [5] using a set of sparse, complex valued antenna index matrices. Repetition of symbols in the DSM encoder was used to achieve diversity gains at the cost of throughput. It subsumes the DSM system of [3] and differential space-time shift keying (STSK) [6], [7] as special cases. Star-QAM STSK was also proposed in [7].

Amplitude phase shift keying (APSK) or star-QAM is known to perform well on fading channels and has been successfully used for differential modulation [7]-[10]. In this letter, we extend the work of [3] to $M-M$ APSK constellations (consisting of $2 M$ points), which can be viewed as two concentric $M$-PSK constellations with different amplitude levels. This is quite different to the STSK approach in [7]. We achieve a spectral efficiency of $\left(N_{t} \log _{2}(M)+\left\lfloor\log _{2}\left(N_{t} !\right)\right\rfloor+N_{t}\right) / N_{t}$ $\mathrm{b} / \mathrm{s} / \mathrm{Hz}$, where the ' $+N_{t}$ ' comes from the choice of amplitude ring of the APSK constellation at each symbol period. This assumes $N_{t}$ transmit antennas and $N_{t}$ symbol periods (only one active antenna per symbol period). We also consider fixing the amplitude level over the DSM matrix resulting in $\left(N_{t} \log _{2}(M)+\left\lfloor\log _{2}\left(N_{t} !\right)\right\rfloor+1\right) / N_{t}$ b/s/Hz. As in [3], no CSI is available at the transmitter or receiver. Our goal is to achieve better throughput than DSM- $M$-PSK and better performance than DSM-2 $M$-PSK using DSM $M$ - $M$ APSK.

Unlike [3], [5], [6], we consider the impact of timevarying fading on performance. As the fading becomes faster, the channel variation during differential detection increases, degrading performance. In [11], the use of reconfigurable antennas and faster fading was shown to improve performance of space-time codes employing long outer error control codes $(E C C s)$, namely low density parity check ( $L D P C)$ codes. In this work, we consider the impact of a long outer ECC on DSM performance. There is a tradeoff between fast fading for the LDPC code and slow fading for DSM detection.

Finally, we investigate using reconfigurable antennas to increase the effective fade rate experienced by the ECC without increasing the fade rate seen by the differential detector. The drawback is a slight reduction in rate due to periodically resending DSM initialization matrices. This approach allows diversity gains and enables the error control decoder to significantly enhance performance. This is quite different to the way diversity is achieved in [5] as our redundancy (protection) is added using a powerful ECC rather than using repetition of symbols in the DSM matrix. Our work on outer codes and reconfigurable antennas in time-varying fading could be applied to the DSM schemes of [3], [5]. In [12], they propose using a single reconfigurable antenna with multiple states in STSK instead of multiple transmit antennas for transmission. In contrast, we use multiple reconfigurable antennas at both transmitter and receiver in order to vary the channel over the length of the outer ECC.

This letter is organised as follows. Section II describes the DSM-PSK scheme of [3], our proposed DSM-APSK scheme, the outer ECC and the proposed reconfigurable antenna switching scheme. In Section III simulation results are given and conclusions are drawn in Section IV.

\section{DifFerential Spatial Modulation (DSM)}

\section{A. DSM-PSK}

The differential transmission matrix for DSM-PSK [3] is defined by

$$
\boldsymbol{S}(\tau+1)=\boldsymbol{S}(\tau) \boldsymbol{X}(\tau+1)
$$

where $\boldsymbol{S}(0)$ is a known $N_{t} \times N_{t}$ initialization matrix spanning $N_{t}$ antennas and $N_{t}$ symbol periods, $\boldsymbol{X}(\tau+1) \in \mathcal{G}$ is selected using $N_{t} \log _{2}(M)+\left\lfloor\log _{2}\left(N_{t} !\right)\right\rfloor$ input bits [4]. The final input 
bit selects the order of antenna use (i.e.. which matrix in $\mathcal{G}$ is employed). For example, for $N_{t}=2$ the set $\mathcal{G}$ is given by [3]

$$
\mathcal{G}=\left\{\left[\begin{array}{cc}
s_{1} & 0 \\
0 & s_{2}
\end{array}\right],\left[\begin{array}{cc}
0 & s_{2} \\
s_{1} & 0
\end{array}\right]\right\} .
$$

where $M$-PSK constellation points $s_{1}, s_{2}$ are selected by $\log _{2}(M)$ bits each.

The received signal matrix for DSM-PSK in [4] over time intervals $N_{t} t$ to $N_{t}(t+1)-1$ is given by

$$
\boldsymbol{Y}(\tau)=\boldsymbol{H}(\tau) \boldsymbol{S}(\tau)+\boldsymbol{N}(\tau)
$$

and over time intervals $N_{t}(t+1)$ and $N_{t}(t+2)-1$ by

$$
\boldsymbol{Y}(\tau+1)=\boldsymbol{H}(\tau+1) \boldsymbol{S}(\tau+1)+\boldsymbol{N}(\tau+1),
$$

where $\boldsymbol{S}(\tau)$ is the $N_{t} \times N_{t}$ transmitted DSM code matrix, $\boldsymbol{N}(\tau)$ is the $N_{r} \times N_{t}$ additive white Gaussian noise (AWGN) matrix over $N_{t} t$ and $N_{t}(t+1)-1, \boldsymbol{H}(\tau)$ is a $N_{r} \times N_{t}$ fading channel matrix and $N_{r}$ is the number of receive antennas. For quasi-static $(Q S)$ fading $\boldsymbol{H}(\tau+1)=\boldsymbol{H}(\tau)$ is constant over two consecutive matrix transmissions.

In order to be able to consider a time varying fading channel we extend the received signal notation of [4] in (3) to

$$
\boldsymbol{Y}(\tau)=\tilde{\boldsymbol{H}}(\tau) \tilde{\boldsymbol{S}}(\tau)+\boldsymbol{N}(\tau),
$$

where $\tilde{\boldsymbol{H}}(\tau)=\left[\boldsymbol{H}\left(N_{t} t\right), \cdots, \boldsymbol{H}\left(N_{t}(t+1)-1\right)\right], \boldsymbol{H}\left(N_{t} t\right)$ is the $N_{r} \times N_{t}$ channel matrix at time $N_{t} t, \tilde{\boldsymbol{S}}(\tau)=$ $\operatorname{diag}\left(\boldsymbol{s}\left(N_{t} t\right), \cdots, \boldsymbol{s}\left(N_{t}(t+1)-1\right)\right)$, and $\boldsymbol{s}\left(N_{t} t+b\right)$ is the $(b+1)^{t h}$ column vector $\left(N_{t} \times 1\right)$ of $\boldsymbol{S}(\tau), b \in 0, \cdots, N_{t}-1$. The variation between $\boldsymbol{H}\left(N_{t} t\right)$ and $\boldsymbol{H}\left(N_{t}(t+1)-1\right)$ will depend on the fade rate, $f_{D} T$, where $f_{D}$ is Doppler frequency and $T$ is symbol period.

The order that transmit antennas are used in the encoded DSM matrix changes with time. This means a given transmit antenna (and associated sub-channel) will be used again after 1 to $2 N_{t}-1$ symbol periods. The maximum number of symbol periods, $2 N_{t}-1$, quickly increases with $N_{t}$. This increases variation for some sub-channels between consecutive DSM matrices going into the differential detector. Channel variation degrades detection performance. This should be taken into account when designing DSM matrix sets, $\mathcal{G}$, for time varying channels.

The differential detector estimates the data matrix $\boldsymbol{X}(\tau+1)$ using an exhaustive search over $\boldsymbol{s}_{1}$ and $\boldsymbol{s}_{2}$ according to [3]

$$
\hat{\boldsymbol{X}}(\tau+1)=\arg \min _{\forall \hat{\boldsymbol{X}} \in \mathcal{G}}\|\boldsymbol{Y}(\tau+1)-\boldsymbol{Y}(\tau) \hat{\boldsymbol{X}}\|_{F}^{2},
$$

where $\|\cdot\|_{F}$ is the Frobenius norm.

\section{B. DSM-APSK}

Now we extend the DSM-PSK scheme of [3] to APSK modulation, DSM-APSK, based on the differential APSK (DAPSK) methodology ${ }^{1}$ of [10]. We differentially encoded both amplitude and phase. We can think of the $M-M$ APSK constellation of Fig. 1 as two concentric $M$-PSK constellations with respective amplitude levels ${ }^{2}$ of $r_{H}$ and $r_{L}$.

\footnotetext{
${ }^{1}$ Although, the contexts are quite different, the methodology can be applied.

${ }^{2}$ We use a ring ratio $r_{H} / r_{L}=2$ as in [9], [10]. Having the same number of points in each ring makes differential encoding easier. We focus on two levels, but extension beyond this is possible.
}

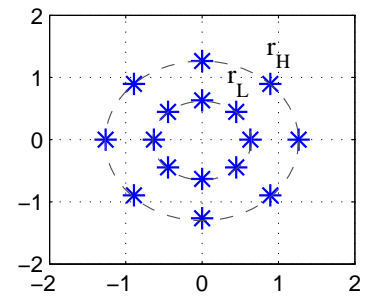

Fig. 1. 8-8 APSK constellation $\left(r_{L}=0.632, r_{H}=1.265\right.$ and $\left.E_{s}=1\right)$.

Method 1: Constant M-M APSK amplitude level over $\boldsymbol{S}(\tau)$. This offers $\left(N_{t} \log _{2}(M)+\left\lfloor\log _{2} N_{t} !\right\rfloor+1\right) / N_{t} \mathrm{~b} / \mathrm{s} / \mathrm{Hz}$ with $\log _{2}(M)$ bits per M-PSK symbol, $\left\lfloor\log _{2}\left(N_{t} !\right)\right\rfloor$ antenna order bits [4] and 1 APSK level bit.

The simple recursion of (1) must now be extended to include a differential amplitude/ level encoding. Now (1) becomes

$$
\boldsymbol{S}(\tau+1)=a_{\tau} \boldsymbol{S}(\tau) \boldsymbol{X}(\tau+1)
$$

where

$$
a_{\tau}= \begin{cases}1, & \text { if } b_{\text {level }}(\tau)=0 \\ r_{H} / r_{L}, & \text { if } b_{\text {level }}(\tau)=1 \text { and } a_{\tau-1}=r_{L} \\ r_{L} / r_{H}, & \text { if } b_{\text {level }}(\tau)=1 \text { and } a_{\tau-1}=r_{H}\end{cases}
$$

and $b_{\text {level }}(\tau)$ is the bit for block $\tau$ defining the amplitude level. Note we initially send $\boldsymbol{S}(0)$ with amplitude level $r_{L}$.

The differential detector is based on concepts in [3], [10]. We use a modified version of (6), which exhaustively searches over all possible antenna order values, APSK amplitude levels and PSK symbols. The symbols are estimated using

$$
\left\{\hat{\boldsymbol{X}}(\tau+1), \hat{a_{\tau}}\right\}=\arg \min _{\forall \hat{\boldsymbol{X}} \in \mathcal{G}, \forall \hat{a}}\|\boldsymbol{Y}(\tau+1)-\hat{a} \boldsymbol{Y}(\tau) \hat{\boldsymbol{X}}\|_{F}^{2} .
$$

The two possible values of $\hat{a}$ depend on the previous estimate $\hat{a}_{\tau-1}$ (from (8) either $\hat{a} \in\left\{1, r_{H} / r_{L}\right\}$ or $\hat{a} \in\left\{1, r_{L} / r_{H}\right\}$ ).

Method 2: Change the APSK amplitude level for symbols $s_{1}$ through $s_{N_{t}}$ independently using $N_{t}$ bits. This gives throughput $\left(N_{t} \log _{2}(M)+\left\lfloor\log _{2} N_{t} !\right\rfloor+N_{t}\right) / N_{t} \mathrm{~b} / \mathrm{s} / \mathrm{Hz}$ as there are now $N_{t}$ level bits. The transmission matrix of (7) becomes

$$
\boldsymbol{S}(\tau+1)=\boldsymbol{A}(\tau+1) \boldsymbol{S}(\tau) \boldsymbol{X}(\tau+1),
$$

where $\boldsymbol{A}(\tau+1)=\operatorname{diag}\left(a_{1}(\tau+1), \cdots, a_{N_{t}}(\tau+1)\right)$ and each $a_{i}(\tau)$ is selected according to (8). The detector uses

$$
\hat{\boldsymbol{A}}=\left[\begin{array}{cc}
\hat{a}_{1} & 0 \\
0 & \hat{a}_{2}
\end{array}\right]
$$

where $\hat{a}_{i} \in\left\{1, r_{H} / r_{L}\right\}$ if $\hat{a}_{i}(\tau)=r_{L}$ and $\hat{a}_{i} \in\left\{1, r_{L} / r_{H}\right\}$ if $\hat{a}_{i}(\tau)=r_{H}$. The estimated $\hat{\boldsymbol{X}}(\tau+1)$ and $\hat{\boldsymbol{A}}(\tau+1)$ matrices are chosen using

$$
\{\hat{\boldsymbol{X}}(\tau+1), \hat{\boldsymbol{A}}(\tau+1)\}=\arg \min _{\forall \hat{\boldsymbol{X}} \in \mathcal{G}, \forall \hat{\boldsymbol{A}} \mid \hat{a}_{i(\tau)}} \mathcal{M}(\hat{\boldsymbol{A}}, \hat{\boldsymbol{X}}),
$$

where

$$
\mathcal{M}(\hat{\boldsymbol{A}}, \hat{\boldsymbol{X}})=\left\|\boldsymbol{Y}(\tau+1)-\boldsymbol{Y}(\tau) \hat{\boldsymbol{S}}^{+}(\tau) \hat{\boldsymbol{A}} \hat{\boldsymbol{S}}(\tau) \hat{\boldsymbol{X}}\right\|_{F}^{2},
$$

$\hat{\boldsymbol{S}}(\tau)$ is the previously estimated $\boldsymbol{S}(\tau)$ matrix and $\hat{\boldsymbol{S}}^{+}(\tau)$ is the pseudo-inverse of $\hat{\boldsymbol{S}}(\tau)$. Using $\hat{\boldsymbol{X}}(\tau+1)$ and $\hat{\boldsymbol{A}}(\tau+1)$, the $N_{t} \log _{2}(M)+\left\lfloor\log _{2} N_{t} !\right\rfloor+N_{t}$ bits can be determined. 


\section{Outer Error Control Code (ECC)}

Outer ECCs are often added to systems to reduce error rates. We consider a LDPC code followed by a bit interleaver. The interleaved encoded bit stream is fed to the DSM-APSK modulator. In the receiver, we demodulate using the DSMAPSK differential detector before LDPC decoding. However, now we need to find the maximum metric, (13), for the most likely bit found using (12) and the competing bit value. Then a scaled approximate log-likelihood ratio $(L L R)$ is calculated (using the max-log MAP assumption) as

$$
L L R_{i}=\frac{\mathcal{M}_{i, 1}-\mathcal{M}_{i, 0}}{2 \sigma^{2}}
$$

where $\sigma^{2}$ is the AWGN variance and $\mathcal{M}_{i, b}$ is the best metric calculated in (12) and (13) with the $i^{t h}$ encoded interleaved bit equal to $b \in\{0,1\}$. Note that a bit ' 0 ' is most likely when $L L R_{i}$ is positive and large. The LLR, then needs to be deinterleaved before being passed to the LDPC decoder. The interleaving is used to break up any error bursts due to the channel or error propagation (from the differential detector). If the parity checks fail, then the LLR of (14) is normalized to a mean of 2 , deinterleaved and re-decoded.

\section{Reconfigurable antennas}

Reconfigurable antennas are able to change the manner in which they radiate, by altering properties such as frequency, polarization and radiation pattern [13], producing different radiation states. Changing the radiation state also changes the channel characteristics experienced in the communication system [13]. It was found in [11] that the performance of a space-time coding scheme employing long ECCs was able to achieve the superior performance of faster fading by periodically or adaptively switching channel conditions (antenna state) over the length of the LDPC codeword. They assumed perfect CSI, while we have no CSI available. Therefore, we propose switching all antenna states at either the transmitter or receiver (or both) $S W$ times during the transmission of an LDPC codeword. This is done in a pre-determined manner (timing and order of states). Each time the states change, the channel changes and the DSM initialization matrix is re-sent and differential detection restarts. This only slightly reduces throughput. This work could also be applied to the DSM schemes of [3], [5].

Let us assume the fade rate is $f_{D} T(1)$, but we want the error control decoder to experience channel variation expected of fade rate $f_{D} T(2)$ (assuming the same transmission format). We need to switch antenna states approximately every

$$
T_{c_{\max }}<\frac{0.3}{f_{D} T(2)}
$$

symbol periods [11]. We assume $L$ is the number of symbol periods spanned by the outer ECC and that all reconfigurable antenna states are independent (correlation will degrade performance, but still allow gains). We denote the number of reconfigurable transmit and receive antenna states (per antenna) as $S_{t}$ and $S_{r}$, respectively. This allows $S_{t} S_{r}$ independent channel

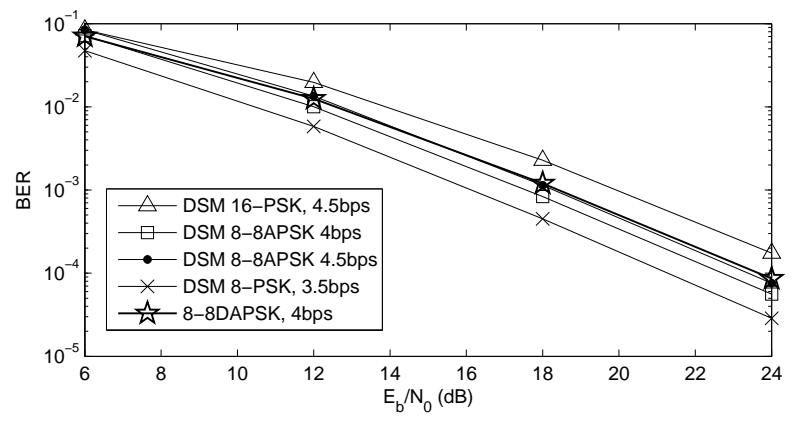

Fig. 2. BER of DAPSK and DSM with PSK [3] and APSK for QS channel.

matrices. The resulting rule of thumb from [11] is to use sufficient states to satisfy

$$
S_{t} S_{r}>\frac{f_{D} T(2) L}{0.3}
$$

This is good for QS fading or an unknown $f_{D} T(1)$. For time varying fading with known $f_{D} T(1)$ we can use the natural variation of the channel (and antenna state reuse) to reduce the number of antenna states required. We can reuse previous antenna states when their conditions should be sufficiently uncorrelated. This results in a more modest $S_{t} S_{r}$ value, but requires a rough estimate of the fade rate to be known at transmitter and receiver. In this case we have

$$
S_{t} S_{r}>\frac{f_{D} T(2)}{f_{D} T(1)}
$$

but change states every $T_{c, \max }$ symbol periods, (15).

\section{Simulation Results}

We now present simulation results for DSM using PSK and APSK. We assume $N_{t}=N_{r}=2$ antennas with $N_{t} N_{r}$ independent sub-channels. We initially consider a QS channel constant over a frame of $N=128$ symbol periods (or 64 DSM matrices) and varying independently between frames. We also consider a time-varying Rayleigh fading channel with fade rate, $f_{D} T$. We plot performance against data bit energy divided by noise spectral density, $E_{b} / N_{0}$.

In Fig. 2 we show the uncoded bit error rate (BER) performance on a QS channel for 8-8 DAPSK (no SM, 4 $\mathrm{b} / \mathrm{s} / \mathrm{Hz})$, DSM 8-PSK $(3.5 \mathrm{~b} / \mathrm{s} / \mathrm{Hz})$ and DSM 16-PSK (4.5 $\mathrm{b} / \mathrm{s} / \mathrm{Hz}$ ) using the scheme of [3], as well as the proposed DSM 8-8APSK ( $4 \mathrm{~b} / \mathrm{s} / \mathrm{Hz}$ for method 1 and $4.5 \mathrm{~b} / \mathrm{s} / \mathrm{Hz}$ for method 2 depending on whether the APSK level changes for each matrix or each symbol). The 8-8APSK curves fall between those for 8-PSK and 16-PSK. However, 8-8APSK has higher throughput than 8-PSK, and better performance than 16-PSK for the same throughput. Method 2 offers higher throughput than method 1 at the cost of performance. DSM APSK offers higher throughput or better performance than DAPSK. This advantage is expected to increase with $N_{t} N_{r}$ [4].

The uncoded BER and frame error rate (FER) for various fade rates are shown in Fig. 3 for DSM 8-8 APSK (4.5 b/s/Hz). For the simulated block length $(N=128)$, the $f_{D} T=0.001$ and QS results are similar. As the fade rate becomes faster, 


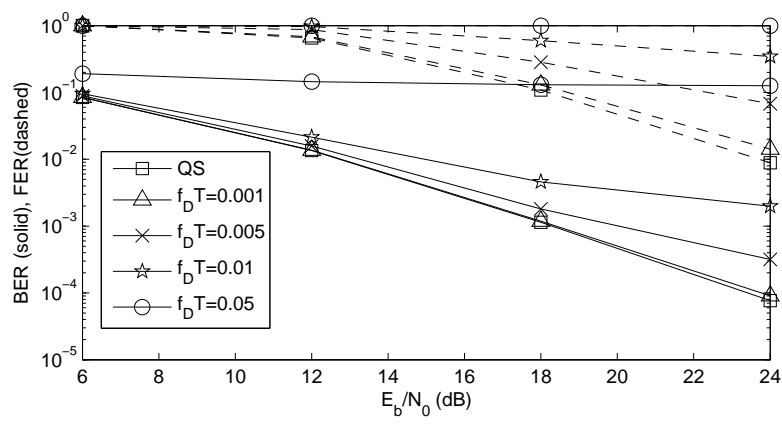

Fig. 3. BER and FER of DSM 8-8APSK method 2 for QS and time varying fading channels.

the channel variation degrades high $E_{b} / N_{0}$ performance of the differential detector. By $f_{D} T=0.05$ it is unusable.

Now we look at the performance for a long outer ECC with and without reconfigurable antennas. We consider a powerful (4096, 1844) LDPC code spanning 455 DSM matrices ${ }^{3}$ ( $L=$ 910 and overall system rate $2.02 \mathrm{~b} / \mathrm{s} / \mathrm{Hz}$ ), but other codes could be used. As expected [11], without reconfigurable antennas the coded DSM results show little improvement over uncoded DSM for slow fading due to long error bursts over the LDPC codeword. In addition, results are poor in fast fading due to the differential detection errors.

We now incorporate reconfigurable antennas and switch antenna states $S W=16$ (or 32) times over the LDPC code transmission (meaning $f_{D} T(2) \approx 0.005$ (or 0.01 ), $S_{t} S_{r}=16$ (or 32 ) and the channel changes every $56-58$ (or $28-30$ ) symbol periods). The initial DSM matrix, $\boldsymbol{S}(0)$, is sent at each switch and differential detection is restarted. The overall rate becomes 1.96 (or 1.89 )b/s/Hz. As can be seen in Fig 4, there is a significant slope gain from changing channel conditions (using reconfigurable antennas) over the length of the LDPC code $(S W=16$ or 32$)$. This agrees with findings in [11], but for the very different case of differential encoding. A criticism of reconfigurable antenna schemes has been the increased channel estimation requirements, but the use of differential encoding and detection avoids this. Simply reinitializing with no state change (16init) can provide most of the gain of $S W=16$ when $f_{D} T(1)=f_{D} T(2)=0.005$, but significantly less gain for slower channels (eg. $f_{D} T(1)=0.001$ ).

\section{CONCLUSIONS}

We proposed a new DSM-APSK scheme, which improved performance or increased throughput compared to DSM-PSK. We also investigated the degradation in DSM performance with increasing fade rate. In addition, we considered the impact of an outer ECC. The tradeoff between fade rate for detection and ECC decoding was considered in detail. A reconfigurable antenna scheme was proposed to increase channel variation over the length of the ECC. This significantly improved performance at the cost of complexity and throughput. The $N_{t}>2$ case is part of ongoing research.

${ }^{3}$ See [11] for details on the LDPC code. Only 4095 bits are required for the 455 DSM matrices, meaning one bit is not transmitted. Maximum of 100 decoding iterations, with early termination when a codeword is found.
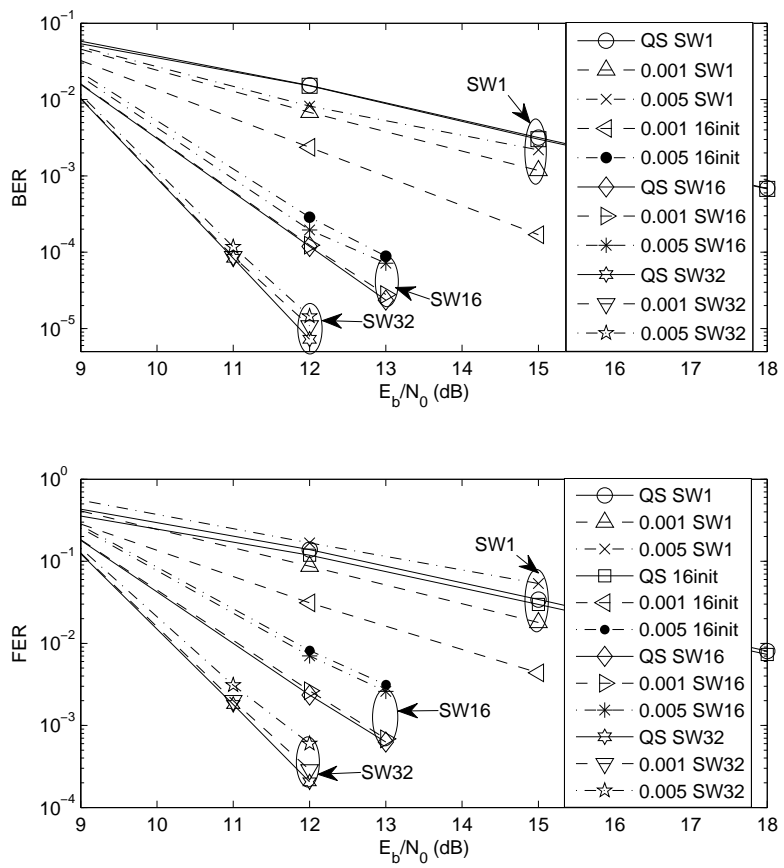

Fig. 4. BER and FER for DSM 8-8APSK method 2, (4096, 1844) LDPC code with $(S W=16,32)$ and without $(S W=1)$ reconfigurable antenna switching. "16init" uses non-reconfigurable antennas with 16 reinitializations.

\section{REFERENCES}

[1] M. Di Renzo, H. Haas and P. M. Grant, "Spatial Modulation for MultipleAntenna Wireless Systems: A Survey", IEEE Commun. Mag., vol. 49, no. 12, pp. 182-191, Dec. 2011.

[2] M. Di Renzo, H. Haas, A. Ghrayeb, S. Sugiura and L. Hanzo, "Spatial Modulation for Generalized MIMO: Challenges, Opportunities and Implementation", Proc. IEEE, vol. 102, no. 1, pp. 56-103, Jan. 2014.

[3] Y. Bian, M. Wen, X. Cheng, H. V. Poor and B. Jiao, "A Differential scheme for spatial modulation", in Proc. Globecom, 2013.

[4] Y. Bian, X. Cheng, M. Wen, L. Yang, H. V. Poor and B. Jiao, "Differential spatial modulation", to appear in IEEE Trans. Veh. Tech., 2014.

[5] N. Ishikawa and S. Sugiura, "Unified Differential Spatial Modulation", IEEE Wireless Commun. Lett., vol. 3, no. 4, pp. 337-340, Aug. 2014.

[6] S. Sugiura, S. Chen and L. Hanzo, "Coherent and differential space-time shift keying: A dispersion matrix approach", IEEE Trans. Commun., vol. 58, no. 11 , pp. 3219-3230, Nov. 2010.

[7] S. Sugiura, C. Xu, S. X. Ng and L. Hanzo, "Reduced-complexity coherent versus non-coherent QAM-aided space-time shift keying", IEEE Trans. Commun., vol. 59, no. 11, pp. 3090-3101, Nov. 2011.

[8] F. Adachi and M. Sawahashi, "Decision feedback differential detection of differentially encoded 16APSK signals", IEEE Trans. Commun., vol. 44, no. 4, pp. 416-418, Apr. 1996.

[9] Y. C. Chow, A. R, Nix and J. P. McGeehan, "Analysis of 16-APSK modulation in AWGN and Rayleigh fading channel", Electron. Lett., vol. 28, no. 17, pp. 1608-1610, 13th Aug. 1992.

[10] X.-G. Xia, "Differentially en/decoded orthogonal space-time block codes with APSK signals", IEEE Commun. Lett., vol. 6, no. 4, pp. 150-152, Apr. 2002.

[11] P.A. Martin, P. J. Smith and R. Murch, "Improving Space-Time Code Performance in Slow Fading Channels using Reconfigurable Antennas", IEEE Commun. Lett., vol. 16. no. 4, pp. 494-497, Apr. 2012.

[12] S. Sugiura, "Coherent versus non-coherent reconfigurable antenna aided virtual MIMO systems", IEEE Signal Proc. Lett., vol. 21, no. 4, pp. 390394, Apr. 2014.

[13] A. Grau, H. Jafarkhani and Franco De Flaviis, "A reconfigurable multiple-input multiple-output communication system," IEEE Trans. Wireless Commun., vol. 7, no. 5, pp. 1719-1733, May 2008. 